\title{
Adipocyte LDL receptor-related protein-1 expression modulates postprandial lipid transport and glucose homeostasis in mice
}

\author{
Susanna M. Hofmann, ${ }^{1}$ Li Zhou, ${ }^{2}$ Diego Perez-Tilve, ${ }^{3}$ Todd Greer, ${ }^{1}$ Erin Grant, ${ }^{1}$ \\ Lauren Wancata, ${ }^{1}$ Andrew Thomas, ${ }^{1}$ Paul T. Pfluger, ${ }^{3}$ Joshua E. Basford, ${ }^{1}$ \\ Dean Gilham, ${ }^{1}$ Joachim Herz, ${ }^{2}$ Matthias H. Tschöp, ${ }^{3}$ and David Y. Hui ${ }^{1}$
} 1Department of Pathology, University of Cincinnati College of Medicine, Cincinnati, Ohio, USA. 2Department of Molecular Genetics, University of Texas
Southwestern Medical Center, Dallas, Texas, USA. ${ }^{3}$ Department of Psychiatry, University of Cincinnati College of Medicine, Cincinnati, Ohio, USA.

\begin{abstract}
Diet-induced obesity and its serious consequences such as diabetes, cardiovascular disease, and cancer are rapidly becoming a major global health threat. Therefore, understanding the cellular and molecular mechanisms by which dietary fat causes obesity and diabetes is of paramount importance in order to identify preventive and therapeutic strategies. Increased dietary fat intake results in high plasma levels of triglyceride-rich lipoproteins (TGRL). Tissue uptake of TGRL has been shown to promote glucose intolerance. We generated mice with an adipocyte-specific inactivation of the multifunctional receptor LDL receptor-related protein-1 (LRP1) to determine its role in mediating the effects of TGRL on diet-induced obesity and diabetes. Knockout mice displayed delayed postprandial lipid clearance, reduced body weight, smaller fat stores, lipid-depleted brown adipocytes, improved glucose tolerance, and elevated energy expenditure due to enhanced muscle thermogenesis. We further demonstrated that inactivation of adipocyte LRP1 resulted in resistance to dietary fat-induced obesity and glucose intolerance. These findings identify LRP1 as a critical regulator of adipocyte energy homeostasis, where functional disruption leads to reduced lipid transport, increased insulin sensitivity, and muscular energy expenditure.
\end{abstract}

\section{Introduction}

The chronic consumption of meals rich in fat and carbohydrates is a major causative factor of obesity and diabetes. The prevailing view on the mechanism by which these dietary factors contribute to obesity and diabetes is that when energy intake surpasses expenditure, the excess calories are deposited as fat in adipose tissues and its subsequent mobilization to nonadipose tissues causes insulin resistance that ultimately leads to type 2 diabetes (1-3). High fat and carbohydrate intake also leads to plasma lipid abnormalities, including high plasma levels of nonesterified fatty acids (NEFAs) and triglyceride-rich lipoproteins (TGRL) as well as reduced plasma levels of $\operatorname{HDL}(2,3)$. Numerous past studies have shown that elevated plasma NEFA levels directly induce insulin resistance and thus play a causative role in the pathogenesis of obesity-related diabetes $(1,4,5)$. However, only sporadic attention has been paid to the role of TGRL in obesity and diabetes. The TGRL are generally thought of primarily as triglyceride carriers in the circulation, delivering substrates to tissues where lipoprotein lipase-catalyzed (LpL-catalyzed) hydrolysis liberates NEFAs prior to their uptake by cells through CD36 and other pathways (6). It is important to note that TGRL as well as lipase-hydrolyzed TGRL remnants can also be internalized by cells directly via whole-particle uptake and provide triglyceride-derived fatty acids via mechanisms mediated by LDL receptor family member proteins (7). Results showing

Nonstandard abbreviations used: BAT, brown adipose tissue; BW, body weight; LpL, lipoprotein lipase; LRP1, LDL receptor-related protein-1; NEFA, nonesterified fatty acid; TGRL, triglyceride-rich lipoprotein(s); UCP-1, uncoupling protein-1; WAT, white adipose tissue.

Conflict of interest: The authors have declared that no conflict of interest exists. Citation for this article: J. Clin. Invest. 117:3271-3282 (2007). doi:10.1172/JCI31929. that $A p o e^{-/-}$mice with defects in receptor-mediated tissue uptake of TGRL are less susceptible to diet-induced obesity and diabetes suggested the importance of TGRL whole-particle uptake in dietinduced obesity and diabetes (8). Indeed, a recent study reporting that TGRL uptake by skeletal muscle cells, independent of NEFA uptake, also directly modulates glucose metabolism and insulin sensitivity is supportive of this hypothesis (9).

One mechanism by which apoE modulates diet-induced obesity and diabetes is through its role in lipid transport and lipid redistribution among different cells and tissues. This process is dictated by the expression level and distribution of various apoE-binding receptors on tissues such as the liver, muscle, heart, and adipose tissues. The 3 apoE binding receptors prominently expressed in these tissues are LDL receptor, VLDL receptor, and LDL receptor-related protein-1 (LRP1). Although these receptors share the overlapping functions of binding and internalizing apoE-containing lipoproteins, there are distinct differences in preference for various lipoproteins. The LDL receptor binds remnants of chylomicrons and VLDL as well as LDL but interacts poorly with nascent chylomicrons and VLDL (10-12). The VLDL receptor appears to prefer intermediate-density lipoprotein (IDL) (13) and immobilizes LpL on the cell surface for hydrolysis of the triglycerides prior to fatty acid uptake into cells (14). In contrast, LRP1 prefers to interact with apoE-enriched chylomicron and VLDL remnants $(15,16)$. The latter receptor is also responsible for LpL-mediated endocytosis of whole lipoprotein particles (17-19). Thus, whereas VLDL receptor expression in high-energy metabolism tissues may modulate sensitivity to diet-induced obesity and diabetes via LpL-catalyzed fatty acid uptake $(14,20)$, the high level of LRP1 expression in adipocytes (21) suggests that LRP1 may partner with apoE and/or LpL in mediating the effects of TGRL in diet-induced obesity. In view of studies establishing the importance of adipose tis- 
A

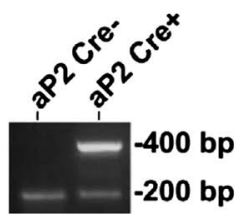

B

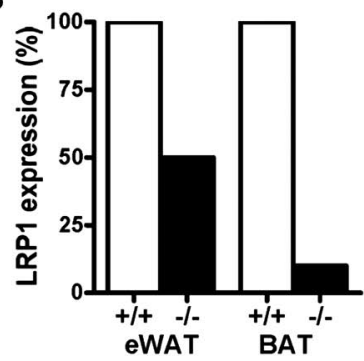

C

\begin{tabular}{|c|lr|c|c|}
\cline { 2 - 5 } \multicolumn{1}{c|}{} & eWAT & BAT & Brain \\
\hline Ad-Lrp1 & $+/+\quad-/-$ & $+/+\quad-/-$ & $+/+-/-$ \\
\hline LRP1 & - & - & $=$ & \\
\hline control & - & - & - \\
\hline
\end{tabular}

D

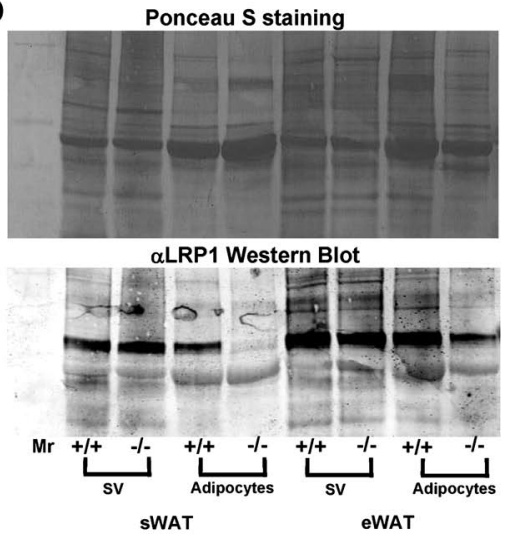

$\mathbf{E}$

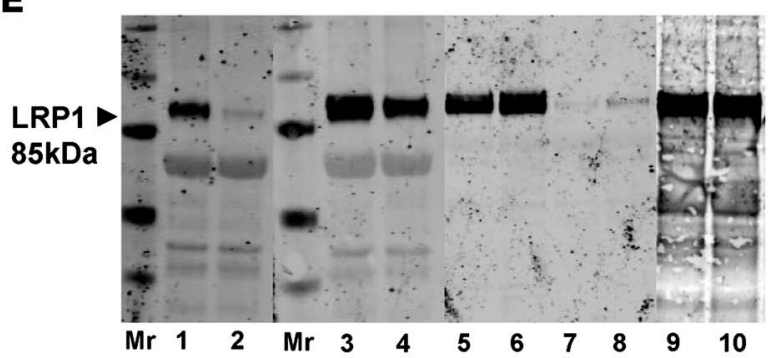

Figure 1

Characteristics of 2 independently generated $a d-L r p 1^{+/+}$and ad-Lrp1-/- mouse colonies. Adipose tissue-specific LRP1 gene inactivation was performed both on a mixed background (Dallas) and on an inbred C57BL/6 background (Cincinnati) by crossing aP2 Cre transgenic mice with Lrp1 $1^{\text {flox/flox }}$ mice. (A) Detection of the Cre transgene in mice with the mixed background by PCR. A fragment of $400 \mathrm{bp}$ was amplified from mouse tail genomic DNA when the Cre transgene driven by the aP2 promoter was present. A 200-bp fragment amplified from the aP2 gene was observed in both PCR products as control. (B) Detection of wild-type (+/+) and mutant $(-/-)$ mRNA of mice (mixed background) by quantitative real-time PCR. (C) Western blot identification of the $\beta$ subunit of LRP1 in epididymal WAT (eWAT), BAT, and brain of ad-Lrp1 $1^{+/+}$and ad-Lrp1-/ mice (mixed background). $\beta$-Actin was used as loading control. (D) Ponceau S staining and Western blot of the $\beta$ subunit of LRP1 in the stromal-vascular cells (sv) and mature adipocytes of subcutaneous (sWAT) and eWAT adipose tissue in ad-Lrp1 ${ }^{1^{+/}}$and ad-Lrp1 $1^{-/-}$mice. (E) Western blot of the $\beta$ subunit of LRP1 in BAT (lanes 1 and 2), epididymal WAT (eWAT) (lanes 3 and 4), liver (lanes 5 and 6 ), skeletal muscle (lanes 7 and 8), and peritoneal macrophages (lanes 9, 10) of ad-Lrp1 $1^{+/+}$(lanes 1, 3, 5, 7, and 9) and ad-Lrp1-/- (lanes 2, 4, 6, 8, and 10) mice (inbred background). Molecular size markers (Mr) were applied to the lane flanking the sample lanes as indicated.

sue in controlling glucose homeostasis and energy balance (22-27), we hypothesized that adipocyte expression of LRP1 is also a key modulator of glucose and energy homeostasis. Adipose-specific LRP1knockout mice were produced to test these hypotheses.

\section{Results}

Adipose-specific LRP1 inactivation was achieved independently at both the University of Cincinnati and University of Texas Southwestern Medical Center by crossing Lrp $1^{\text {flox } / \text { flox }}$ mice (28) with aP2-Cre
A

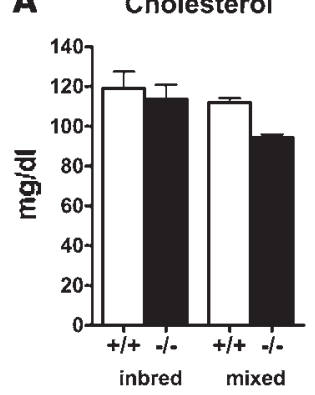

Triglycerides

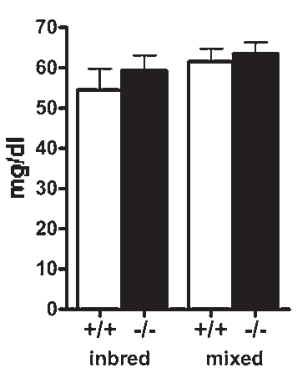

B

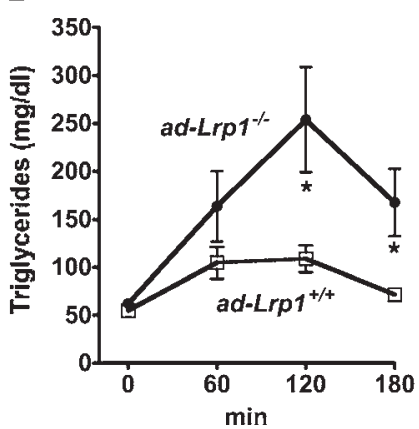

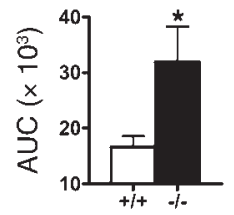

Figure 2

Lipid transport to adipose tissue is impaired in ad-Lrp1-/- mice. (A) Fasting plasma cholesterol and triglyceride levels were similar in ad-Lrp1//+ (open bars) and ad-Lrp1-/- (black bars) mice fed basal chow diet in both inbred and mixed-strain backgrounds. (B) Plasma triglyceride levels in ad-Lrp1 $1^{+/+}$(open squares) and ad-Lrp1 $1^{-/-}$(filled circles) mice after an oral lipid load (inbred background). The inset shows the area under the curve (AUC) analysis of the data. ${ }^{*} P<0.05 ; n=10$. 

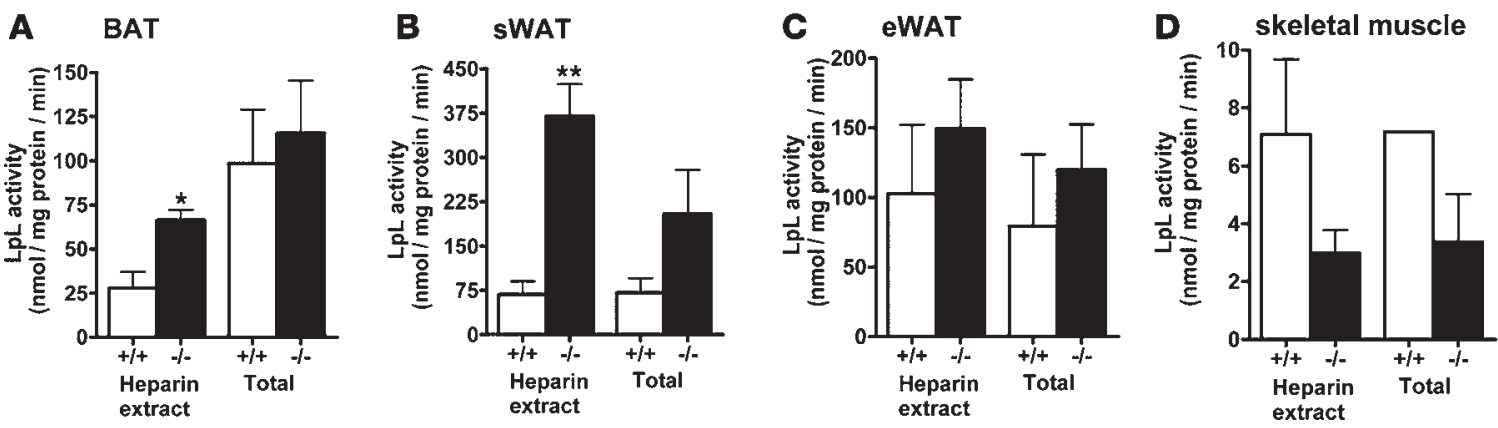

Figure 3

$\mathrm{LpL}$ activities in homogenate (Total) and heparin-releasable (Heparin extract) fractions of BAT (A), sWAT (B), eWAT (C), and skeletal muscle (D) of ad-Lrp1+/+ and ad-Lrp1-/- mice $(n=3) .{ }^{*} P<0.05,{ }^{* *} P<0.005$ compared with the ad-Lrp ${ }^{+/+}$group.

transgenic mice generated in either a homogenous C57BL/6 background (23) or in an outbred strain background (Figure $1 \mathrm{~A})$ to yield ad-Lrp1 $1^{-/-}$mice with either a homogeneous C57BL/6 background or a mixed C57BL/6 and 129 background. In both colonies, the $\operatorname{Lrp} 1$ mRNA level was reduced by approximately $50 \%$ in white adipose tissue (WAT) and by approximately $85 \%$ in brown adipose tissue (BAT) of ad-Lrp1-/- mice compared with their respective ad-Lrp1 $1^{+++}$littermates (Figure 1B). Western blot analysis of LRP1 protein levels in WAT and BAT was consistent with the mRNA expression levels (Figure 1C). When the WAT from subcutaneous (sWAT) and epididymal (eWAT) fat depots of $a d-L r p 1^{+/+}$and ad-Lrp1 $1^{-/-}$mice were fractionated into mature adipocytes and stromal vascular cells, LRP1 expression in WAT of ad-Lrp1 $1^{-/-}$mice was found to be due to its presence at normal levels in the stromal vascular cells (Figure 1D). In contrast, LRP1 expression in mature adipocytes was decreased by greater than $90 \%$ in subcutaneous fat depot and more than $70 \%$ in epididymal fat depot of ad-Lrp $1^{-/-}$mice (Figure 1D). Expression of LRP1 in brain, liver, muscle, and macrophages was not affected in the ad-Lrp1 $1^{-/-}$mice (Figure 1, C and E).

Comparison of $a d-L r p 1^{+/+}$and $a d-L r p 1^{-/-}$mice revealed similar fasting plasma triglyceride and cholesterol levels when the animals were fed a standard low-fat chow diet (Figure 2A). However, postprandial lipid clearance from the circulation in response to a bolus fat load was markedly delayed in $\mathrm{ad}-\operatorname{Lrp}^{1^{-/}}$mice compared with ad-Lrp1 $1^{+/+}$mice (Figure 2B). Since LRP1 binds apoE-containing lipoproteins as well as $\mathrm{LpL}$, the delayed postprandial lipid clearance observed in ad-Lrp $1^{-/}$mice may be due to defective postprandial lipoprotein endocytosis or deficiency in cell-surface LpL that mediates triglyceride hydrolysis prior to cellular fatty acid uptake. These possibilities were explored initially by comparing total and heparin-extractable $\mathrm{LpL}$ activities in various tissues of ad-Lrp $1^{+/+}$and $a d-L r p 1^{-/-}$mice. Results showed no significant difference in LpL activities in total homogenates of BAT and WAT from both subcutaneous and epididymal fat pads and in the muscle of ad-Lrp $1^{+/+}$and ad-Lrp1 $1^{-/-}$mice (Figure 3 ). In contrast, cell-surface $\mathrm{LpL}$, as measured by heparin-releasable $\mathrm{LpL}$ activity, was elevated

\section{Figure 4}

Tissue distribution of radiolabeled lipids after infusion with lipid emulsions containing $\left[{ }^{3} \mathrm{H}\right]$ cholesteryl ester and $\left[{ }^{14} \mathrm{C}\right]$ triolein. Uptake of ${ }^{3} \mathrm{H}(\mathbf{A})$ and ${ }^{14} \mathrm{C}(\mathrm{B})$ labels into BAT, eWAT, muscle, and liver of $\mathrm{ad}-\mathrm{Lrp1} 1^{+/+}$and ad-Lrp1 $1^{-/}$mice $(n=5)$. (C and D) Triglyceride content in liver and muscle, respectively, of ad-Lrp1+/+ and ad-Lrp1-/- mice $(n=8) .{ }^{*} P<0.05$ compared with ad-Lrp1+/+ groups.

significantly in both BAT and WAT (Figure 3). Although decreased heparin-releasable LPL activity was noted in muscle, the difference in heparin-releasable activities from muscle of $a d-L r p 1^{+/+}$and ad-Lrp1 $1^{-/-}$mice did not reach statistical significance (Figure 3). Thus, the delayed postprandial triglyceride clearance in ad-Lrp1 $1^{-/-}$ mice was not due to defective expression of LPL.

The mechanism by which adipocyte LRP1 participates in postprandial lipid clearance were explored by experiments monitoring tissue distribution of dual radiolabels after infusing lipid emulsions containing $\left[{ }^{3} \mathrm{H}\right]$ cholesteryl oleate and $\left[{ }^{14} \mathrm{C}\right]$ triolein into $a d-\operatorname{Lrp} 1^{+/+}$and ad-Lrp1 $1^{-/-}$mice. Results showed that uptake of ${ }^{3} \mathrm{H}$ - and ${ }^{14} \mathrm{C}$-labeled lipids by adipose tissues in ad-Lrp $1^{-/-}$mice was decreased to a similar extent, with the most dramatic decrease observed in BAT (Figure 4). Interestingly, despite the similar level of cholesteryl ester and triglyceride uptake by the liver of $a d-L r p 1^{+/+}$

A

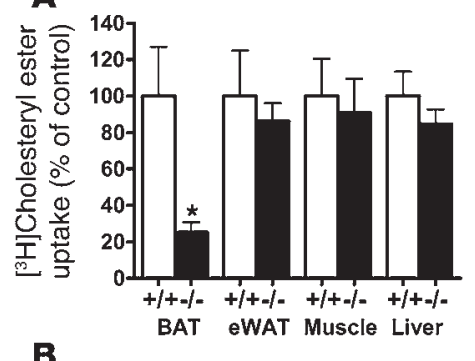

B
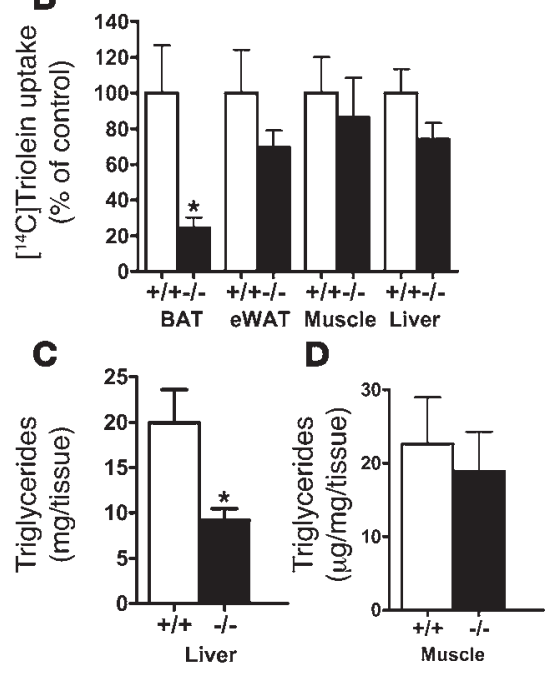
A

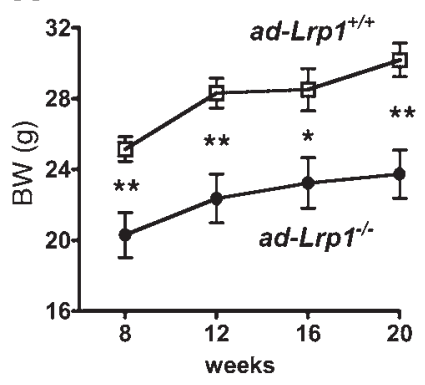

B

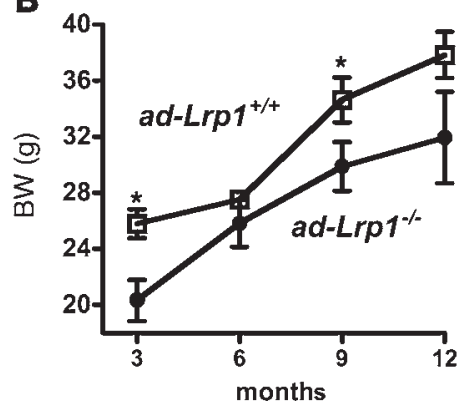

C

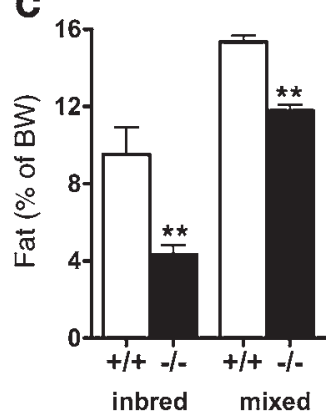

Figure 5

BW and fat mass are reduced in ad-Lrp1 $1^{-/-}$mice. BWs of inbred $(\mathbf{A} ; n=16)$ and mixed $(\mathbf{B} ; n=4)$ ad-Lrp $1^{+/+}$(open squares) and ad-Lrp1 $1^{-/-}$(filled circles) mice. (C) NMR analysis of fat mass content in ad-Lrp1 $1^{+/+}$(white bars) and ad-Lrp1-/- (black bars) mice at 3 month of age (inbred and mixed background). ${ }^{*} P<0.05,{ }^{* *} P<0.005$ compared with the ad-Lrp1 ${ }^{+/+}$groups.

and ad-Lrp1 $1^{-/-}$mice, hepatic triglyceride levels were found to be lower in the ad-Lrp $1^{-/-}$mice (Figure 4C). Triglyceride levels in muscle were similar in ad-Lrp1 $1^{+/+}$and $a d-L r p 1^{-/-}$mice (Figure 4D).

The reduced lipid assimilation by adipocytes in the ad-Lrp1-/mice resulted in lower body weight (BW) as compared with that observed in the ad-Lrp1 $1^{+/+}$mice in both inbred (Figure 5A) and mixed-strain backgrounds (Figure 5B). This BW difference was entirely due to smaller fat mass (Figure 5C), which was also consistent with lower plasma leptin levels observed in the mutant mice $(526 \pm 87 \mathrm{pg} / \mathrm{ml}$ vs. $3,126 \pm 978 \mathrm{pg} / \mathrm{ml}$ in wild type; $P<0.05)$. The reduction of fat mass in $a d-L r p 1^{-/-}$mice was due to a remarkable and macroscopically obvious reduction of interscapular and epididymal fat pads observed in both C57BL/6 and mixed-strain background (Figure 6, A and B). Similarly, BAT mass was reduced by more than $65 \%$ in the ad-Lrp1 $1^{-/-}$mice (Figure 6C). Histological analysis of fat tissues revealed smaller-sized and fewer lipid droplets in white and brown adipocytes in both lines of ad-Lrp1 $1^{-/-}$ mice compared with wild-type mice (Figure 6, D-F). The consis-
A

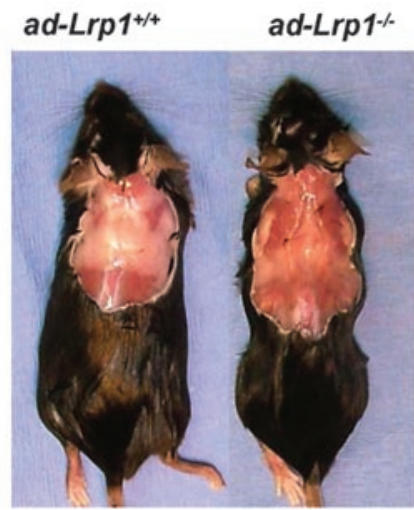

D

D $\quad$ ad-Lrp1 $1^{+/+}$

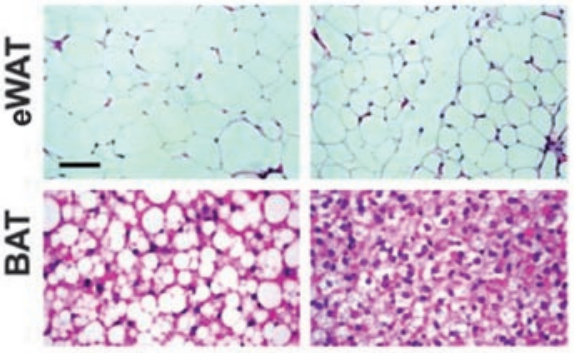

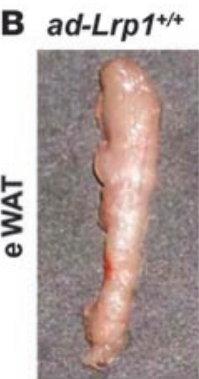
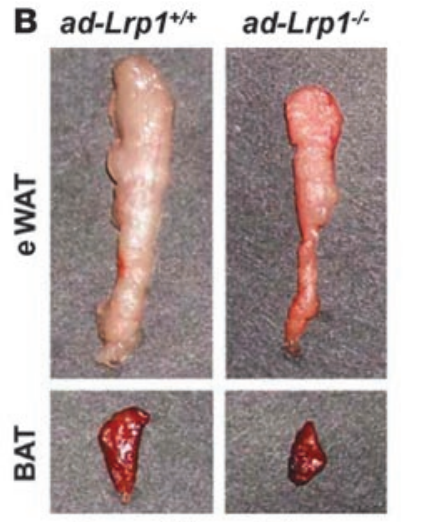

C

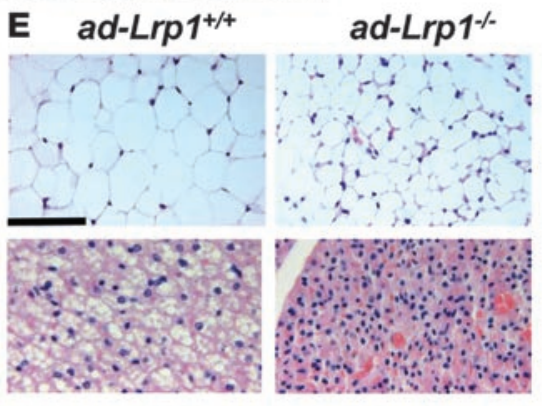

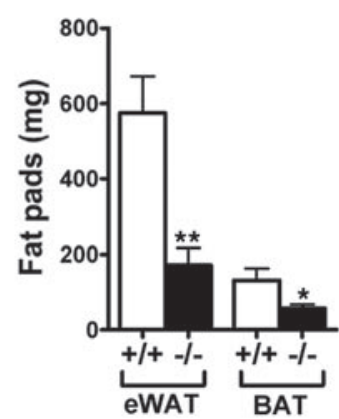

$\mathbf{F}$

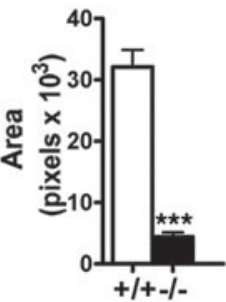

\section{Figure 6}

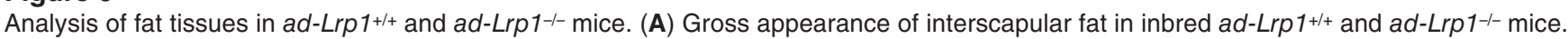
(B) Gross appearance of eWAT fat pads and BAT isolated from ad-Lrp1 $1^{+/+}$and $\mathrm{ad}-\mathrm{Lrp1} 1^{-/-}$mice in a mixed background. (C) eWAT fat pad and BAT mass measurements obtained from inbred ad-Lrp1+/+ (white bars) and ad-Lrp1-/- (black bars) mice $(n=8)$. (D) Histology of eWAT (original magnification, $\times 200$ ) and BAT (original magnification, $\times 400)$ from $a d-L r p 1^{+/+}$and $a d-L r p 1^{-/-}$mice on a mixed background. Scale bar: $25 \mu \mathrm{m}$. (E) Histology of eWAT (original magnification, $\times 400$ ) and BAT (original magnification, $\times 600$ ) from ad-Lrp1 $1^{+/+}$and ad-Lrp1 $1^{-/}$mice in homogeneous C57BL/6 background. Scale bar: $100 \mu \mathrm{m}$. (F) Comparison of adipocyte cell size in ad-Lrp1 $1^{+/+}$and ad-Lrp1 $1^{-/-}$mice as measured by pixel areas. ${ }^{\star} P<0.05,{ }^{* *} P<0.005,{ }^{* *} P<0.001$ compared with the ad-Lrp1 ${ }^{+/+}$group. 

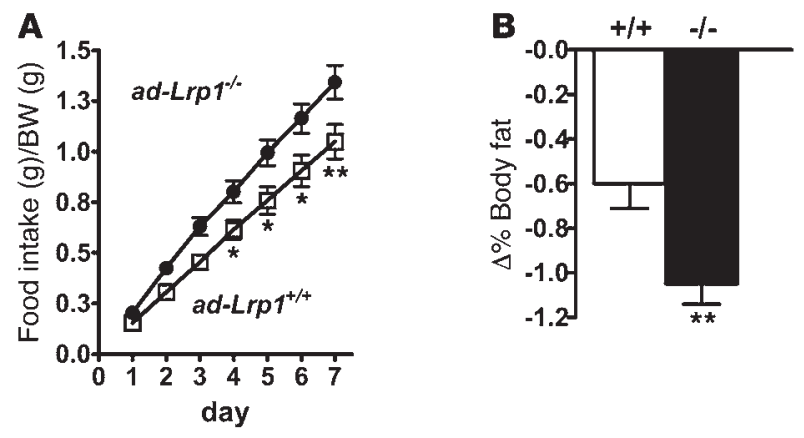

Figure 7

Increased food intake under normal chow-fed conditions and reduced fat mass after food deprivation in ad-Lrp $1^{-/-}$mice. (A) Food intake data collected from inbred ad-Lrp $1^{+/+}$(open squares) and ad-Lrp1 $1^{-/-}$(filled circles) mice $(n=12)$. (B) Changes in fat as a percentage of BW in ad-Lrp1 $1^{+/+}$(white bar) and ad-Lrp1 $1^{-/-}$(black bar) mice on the mixed background after 24 hours of food deprivation $(n=16-18)$. ${ }^{*} P<0.05$, ${ }^{* *} P<0.005$ compared with the ad-Lrp1+/+ groups.

tency of these phenotypes in both inbred and outbred lines of ad-Lrp $1^{-/}$mice underscores the importance and highly penetrant role of LRP1 in the regulation of adipose tissue metabolism. Surprisingly, ad-Lrp1 $1^{-/}$mice were found to consume more food than the ad-Lrp1 $1^{+/+}$mice despite their reduced BW and fat mass (Figure 7A). Moreover, when the animals on the mixed-strain background were fasted for 24 hours, an exaggerated loss of fat mass

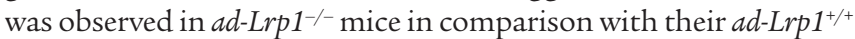
littermates (Figure 7B), which is indicative of an increase in the metabolic rate in the absence of adipocyte LRP1. Consolidating observations on the mixed background with additional studies conducted in the inbred population revealed that all inbred ad-Lrp $1^{-1-}$ mice exhibited difficulty in maintaining body temperature, with increased shivering and body surface reduction by coiling up that was visibly noticeable initially at the ambient environment of $22^{\circ} \mathrm{C}$ and was more pronounced at $4^{\circ} \mathrm{C}$ (see Supplemental Videos 1 and 2; supplemental material available online with this article; doi:10.1172/JCI31929DS1). This was further quantified using a multidimensional light beam system designed to analyze motor activity (Figure 8A). In contrast, ambulatory movement was not altered by LRP1 gene disruption (Figure 8B).

The potential impairment of BAT functions in $a d-L r p 1^{-/-}$mice was further illustrated by results showing that ad-Lrp $1^{+/+}$mice displayed similar body core temperature but significantly lower surface temperature than $a d-\operatorname{Lrp} 1^{+/+}$mice when they were kept at an ambient temperature of $22^{\circ} \mathrm{C}$ (Figure 9). The difference in body surface temperature was further exaggerated when the animals were exposed to a $4{ }^{\circ} \mathrm{C}$ environment (Figure 9). Interestingly, body core temperature also decreased much faster in ad-Lrp $1^{-/-}$mice than ad-Lrp $1^{+/+}$mice when the ambient temperature was lowered (Figure 9).

The increase in muscle shivering activity, decrease in body surface temperature, and the rapid rate of cold-induced core temperature decline may reflect a reduction in resting thermogenesis. Therefore, we examined age-matched $a d-L r p 1^{+/+}$and $a d-L r p 1^{-/-}$mice in an indirect calorimeter to test this hypothesis. While the respiratory quotient was found to be similar (Figure 8C), the mutant mice displayed substantially higher oxygen consumption and energy expenditure (Figure 8D). The increased energy expenditure in mice specifically lacking LRP1 in adipocytes was not due to differences in uncoupling protein-1 (UCP-1) expression in BAT or UCP-2 and UCP-3 expression in muscle (Figure 8E). Thus, the unexpected increased energy expenditure in ad-Lrp1 $1^{-/-}$mice, which could explain their lower fat mass, may reflect at least in part a potential compensatory increase in muscular contractile activity due to the dramatic decrease of BAT.

Numerous mouse models with modest reduction in adiposity and lower leptin levels have been shown to be more insulin sensitive with improved glucose metabolism in vivo $(22,29-31)$. However, other mouse models with reduced adipose tissues such as PPAR $\gamma 2$ knockout mice have impaired insulin sensitivity (32). Therefore, we tested the effects of reduced adipose tissue mass and leptin levels in glucose homeostasis in the ad-Lrp1 $1^{-/-}$mice. Results showed that the ad-Lrp $1^{-/-}$mice have markedly lower fasting glucose and insulin levels compared with wild-type mice (Figure 10A). Lower fasting NEFA levels were also observed in inbred ad-Lrp1 $1^{-/-}$mice (Figure 10A). Glucose tolerance tests showed that the mutant mice also displayed an improved response to glucose challenge (Figure 10B). Tissue distribution of 2-deoxy- $\left[{ }^{3} \mathrm{H}\right]$ glucose revealed that the skeletal muscle was responsible for the elevated plasma glucose clearance in ad-Lrp1 $1^{-/-}$mice (Figure 10C). This observed increase in glucose uptake by skeletal muscle of $a d-L r p 1^{-/-}$mice is consistent with the increased muscular activity in these animals.

The reduced adipose tissue mass along with increased metabolic rate and improved glucose tolerance in ad-Lrp1 $1^{-/}$mice suggested that adipose-specific LRP1 inactivation may be protective against diet-induced obesity and diabetes. This hypothesis was examined by placing $a d-L r p 1^{+/+}$and $a d-L r p 1^{-/-}$mice on a high-fat diet containing 35.8\% fat. Results showed that both ad-Lrp1 $1^{+/+}$and $a d-L r p 1^{-/-}$ mice displayed reduced respiratory quotients under the high-fat feeding conditions and that the difference in energy expenditure between $a d-L r p 1^{1^{-/}}$and $a d-L r p 1^{+/+}$mice was exaggerated (Figure 11, A and B). It is likely that the relative decrease in dietary carbohydrates further impairs the thermogenic efficiency in adLRP1-/mice, with both BAT and muscle metabolism even more dependent on the ability to use lipids. Importantly, the substantially elevated energy expenditure in ad-Lrp $1^{-/-}$mice prevented most of the highfat diet-induced BW gain and adiposity observed in their ad-Lrp1 $1^{+/+}$ littermates (Figure 11, C and D). Finally, in accordance with their protection against diet-induced obesity - and in contrast to wild-type mice, which displayed elevated fasting plasma glucose and insulin levels upon high-fat feeding - plasma glucose and insulin levels in the ad-Lrp $1^{-/-}$mice remained at the normal level after feeding the high-fat diet for 16 weeks (Figure 12, A and B). Glucose tolerance in high-fat diet-fed $\operatorname{ad-Lrp1^{-/-}}$ mice was also similar to that observed in chow-fed mice, while ad-Lrp $1^{+/+}$mice displayed delayed glucose clearance (Figure 12C).

\section{Discussion}

Here we showed that in the absence of LRP1, TGRL assimilation is impaired in adipose tissue, resulting in decreased fat mass. The data also revealed that glucose metabolism and energy balance are enhanced in ad-Lrp $1^{-/-}$mice. The metabolic alterations we have reported with male mice were also evident, albeit less robust, in female mice (data not shown). Furthermore, these phenotypic manifestations due to the loss of LRP1 expression in adipose tissues were essentially identical in mice on a homogenous C57BL/ 6 background as well as on a mixed-strain background, thus documenting the importance and highly penetrant role of adipocyte LRP1 in the regulation of adipocyte tissue 


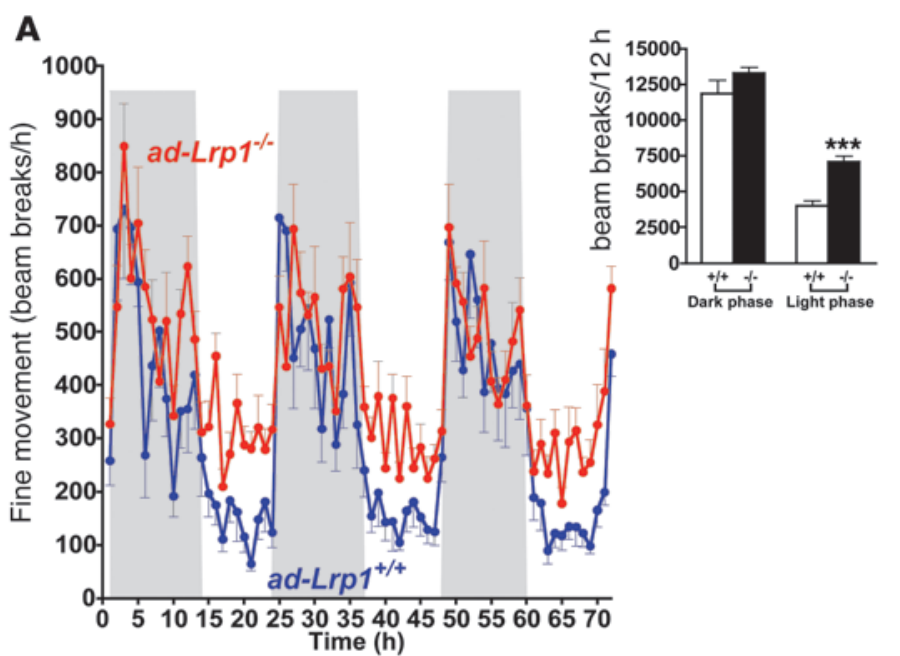

B
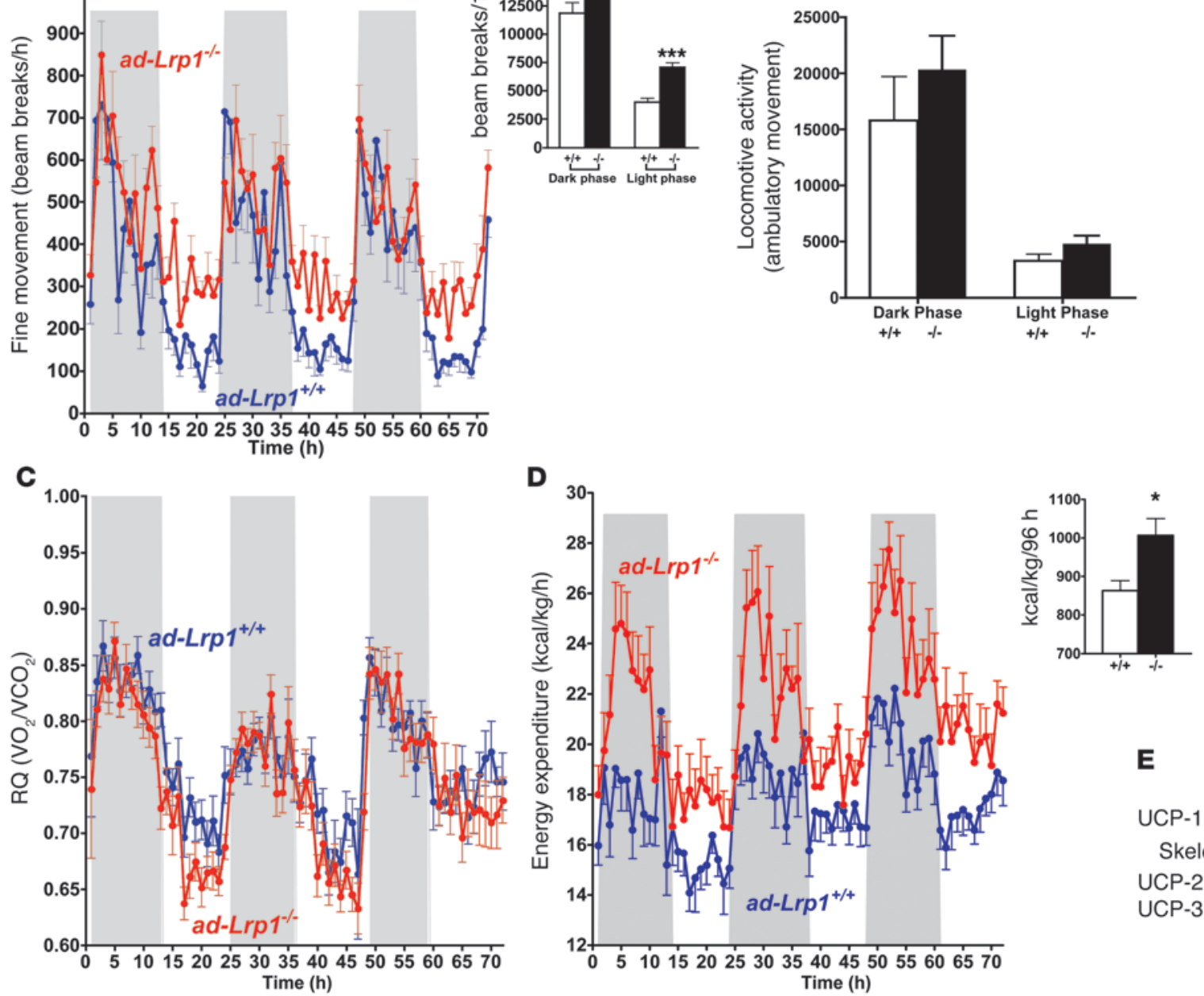

Figure 8

Increased energy expenditure and muscle shivering in ad-Lrp1-1- mice under normal chow-fed conditions. (A) Muscle shivering, or fine stationary motor activity, as detected by breaks of the same light beam set in a metabolic cage, where parallel beams were set up close enough so that each mouse had at least 1 light beam being broken by its body. (B) Cumulative ambulatory movement as measured by breaking of consecutive beams over a 12-hour dark/12-hour light period. Respiratory quotient (C) and energy expenditure (D) were recorded in inbred ad-Lrp1+/+ (blue tracings) and ad-Lrp1-/- (red tracings) mice. The insets in A and D show cumulative fine movement activity and energy expenditure measurements from the respective tracings. Data represent mean \pm SEM from 16 mice per group. (E) Western blots of UCP-1 expression in BAT, UCP-2, and UCP-3 in skeletal muscle of inbred ad-Lrp1 $1^{+/+}$(lane 1) and ad-Lrp1-/- (lane 2) mice. ${ }^{*} P<0.05$ and ${ }^{* * *} P<0.0005$.

metabolism. The aP2 gene promoter commonly used to generate fat tissue-specific knockout and transgenic mouse models $(25,26,33)$ was used in the current study to drive Cre recombinase transgenic expression in $\operatorname{Lrp} 1^{\text {flox/flox }}$ mice for the generation of adipose-specific LRP1-knockout mice. The aP2-Cre recombinase transgene had no effect on LRP1 expression in brain, liver, and macrophages. However, LRP1 expression levels were reduced by approximately $85 \%$ in BAT and by approximately $75 \%-90 \%$ in mature adipocytes of ad-Lrp $1^{-/-}$mice compared with those observed in ad-Lrp1 $1^{+/+}$mice. These findings are consistent with the high LRP1 expression levels reported in nondifferentiated 3T3-L1 preadipocytes and murine embryonic fibroblasts $(34,35)$ and the expected expression of the aP2 promoter-driven Cre recombinase transgene during the late phase of adipocyte differentiation (36).
Results of the current study revealed the importance of adipocyte LRP1 in postprandial TGRL metabolism. Previously, the rate of plasma triglyceride accumulation after a high-fat meal was thought to be dictated by the rate of LpL-mediated triglyceride hydrolysis and the subsequent cellular uptake of the NEFAs (37), as well as receptor-mediated uptake of remnant lipoproteins $(38,39)$. Turnover studies have shown that the liver is the main site of chylomicron remnant clearance and that this process is mediated by the LDL receptor and LRP1 (38-40). Both receptors are also expressed almost ubiquitously in other tissues. Results of the current study showed that the absence of LRP1 in adipose tissues of ad-Lrp $1^{-/-}$mice resulted in delayed postprandial TGRL clearance as well as decreased uptake of both cholesteryl esters and triglycerides from lipid emulsions injected into the circulation. Interestingly, adipose tissue uptake of both lipid moieties was 
A
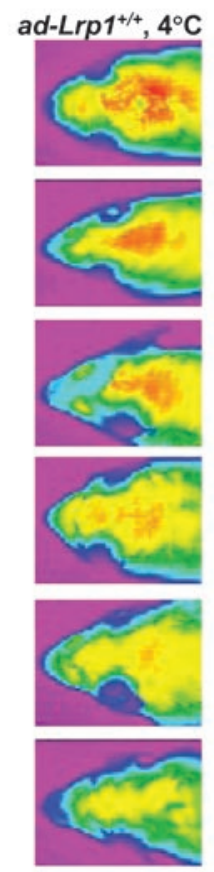
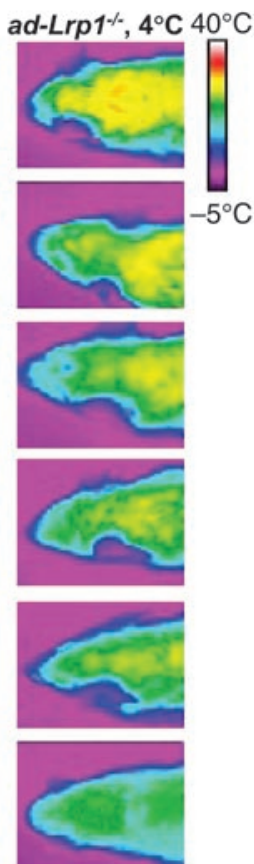

ad- $\operatorname{Lrp1} 1^{+/+}, 22^{\circ} \mathrm{C}$
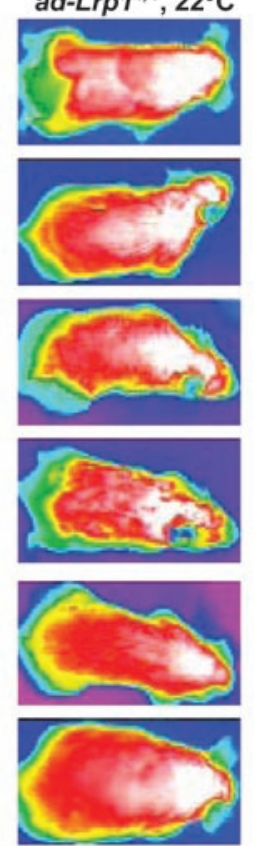

ad-Lrp1\%, $22^{\circ} \mathrm{C} 37^{\circ} \mathrm{C}$
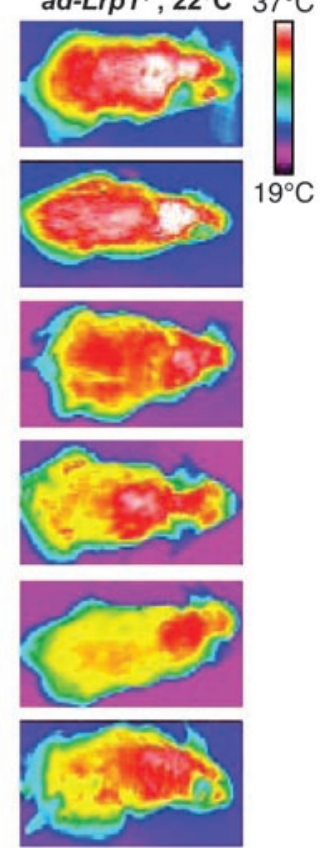

B
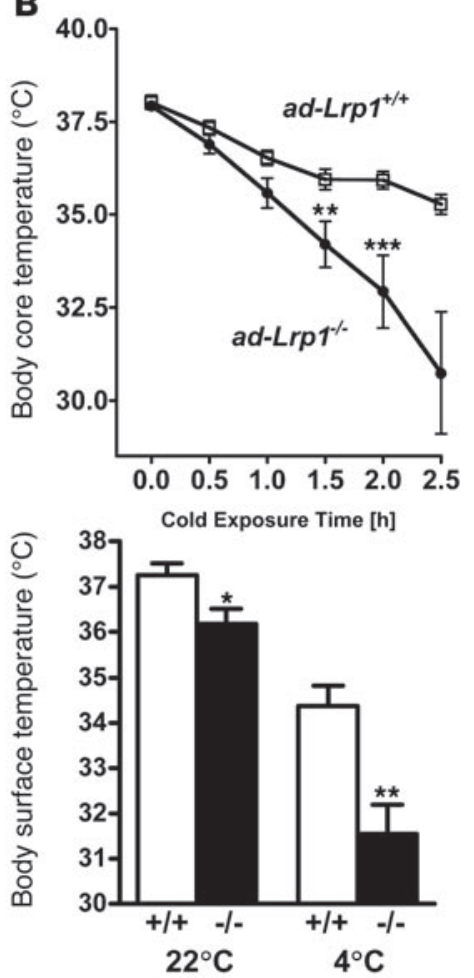

Figure 9

Cold adaptation studies in ad-Lrp1 ${ }^{+/+}$and ad-Lrp1-/- mice. (A) Heat production visualized by a high-resolution infrared camera from 6 mice in each group. (B) Body core and surface temperature measurements from ad-Lrp1 $1^{+/+}$and ad-Lrp1 $1^{-/-}$mice $(n=10) .{ }^{\star} P<0.05,{ }^{\star \star} P<0.005,{ }^{\star \star \star} P<0.001$.

decreased to a similar extent even though heparin-releasable LpL was elevated in adipose tissues of ad-Lrp1 $1^{-/}$mice. Taken together, these results indicated that LRP1 is important in mediating both the lipolytic and the endocytic processes responsible for TGRL catabolism in adipocytes. This interpretation is consistent with previous in vitro data showing a role of LRP1 in mediating LpL binding and lipolysis of TGRL at the cell surface (41), as well as its well-documented role in binding and uptake of LpL- and/or apoE-enriched lipoproteins $(15,21,42)$. It is noteworthy, however, that fasting triglycerides were maintained at the normal levels in ad-Lrp $1^{-1-}$ mice despite their delay in clearance of postprandial TGRL. These data are supportive of previous reports demonstrating that LRP1 is important for clearance of the intestinally derived apoB48-containing lipoproteins but not the catabolism of endogenously derived apoB100-containing lipoproteins (43).

In addition to the delay in postprandial TGRL clearance and alteration in lipid delivery to target tissues, the ad-LRP1-/- mice were also shown to have an overall decrease in fat mass and were protected from high-fat diet-induced glucose intolerance and obesity. Previous studies have clearly established the role of BAT in energy expenditure through thermogenesis in the form of heat dissipation $(44,45)$. Hence, the lower body-surface temperature (less heat dissipation) and increased sensitivity to cold-induced body

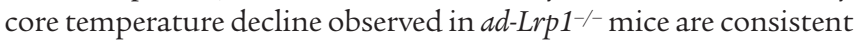
with the reduced BAT mass observed in these animals. Although it has been shown that a complete absence of BAT function is associated with obesity (46), the ad-Lrp1 $1^{-/-}$mice described here are more resistant to diet-induced obesity and more glucose tolerant than wild-type mice. We concluded that the different phenotype of the ad-Lrp1 $1^{-/-}$mice is due to loss of a specific function in all fat depots in contrast to the effect of complete BAT ablation. Similarly, the metabolic phenotypes of the ad-Lrp1 $1^{-/-}$mice are also different from those of the Cidea-deficient mice, which are resistant to diet-induced obesity and diabetes but are more resistant to coldinduced body core temperature drop (47). The difference between ad-Lrp1 $1^{-/-}$and Cidea ${ }^{-/-}$mice is that Cidea is expressed only in BAT, where its major role is to suppress UCP-1 activity (47), whereas LRP1 is normally expressed and mediates lipid uptake in both BAT and WAT. Thus, the lack of Cidea in $\mathrm{Cidea}^{-/-}$mice protects against diet-induced obesity and diabetes due to their elevated energy expenditure as a consequence of increased thermogenic activity in BAT. The constitutively activated BAT thermogenic activity also protects $\mathrm{Cidea}^{-/-}$mice from cold-induced body core temperature decline. In contrast, the reduced BAT mass in ad-Lrp1 $1^{-/-}$ mice results in less heat dissipation and rapid cold-induced body core temperature drop. The resistance of ad-Lrp1 $1^{-/-}$mice against diet-induced obesity is enhanced by additional mechanisms, most likely including the lack of LRP1-mediated lipid delivery to white adipocytes and the increased muscular activity required for the maintenance of body core temperature. It is also possible, and not necessarily mutually exclusive, that LRP1 deletion in adipocytes alters the production of an uncharacterized adipokine that is important for energy expenditure regulation.

The requirement for increased muscular activity in body core temperature maintenance in the ad-Lrp1 $1^{-/-}$mice was supported by observations of their muscle shivering, especially during the light phase, when the animals rest and ambulatory activity is minimal. Such an alternative heating mechanism is substantially less effi- 
A

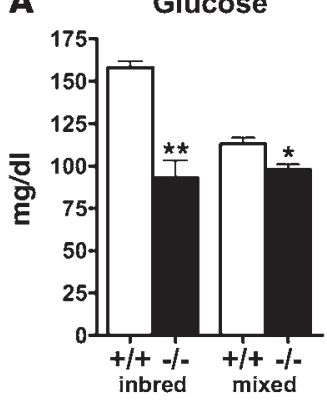

Insulin

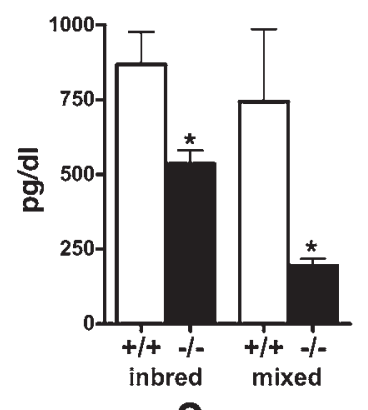

NEFA

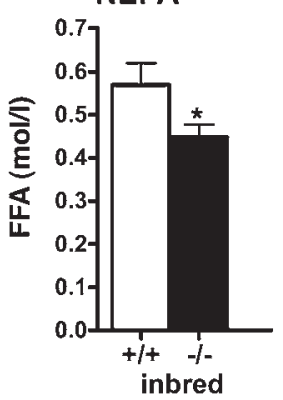

B

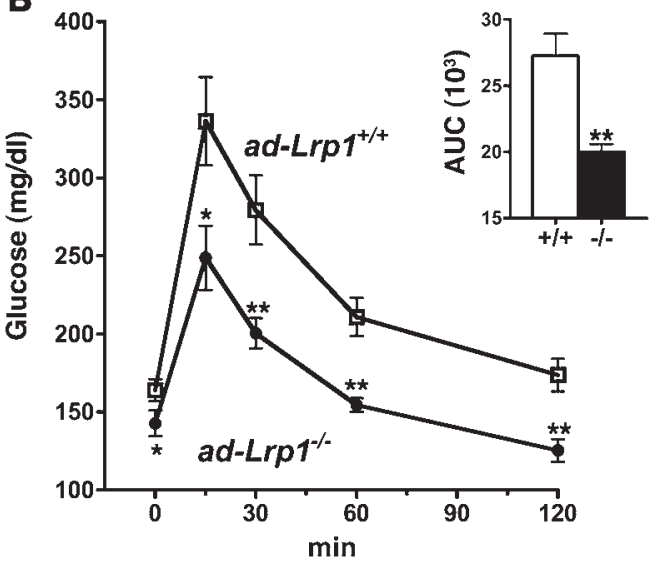

C

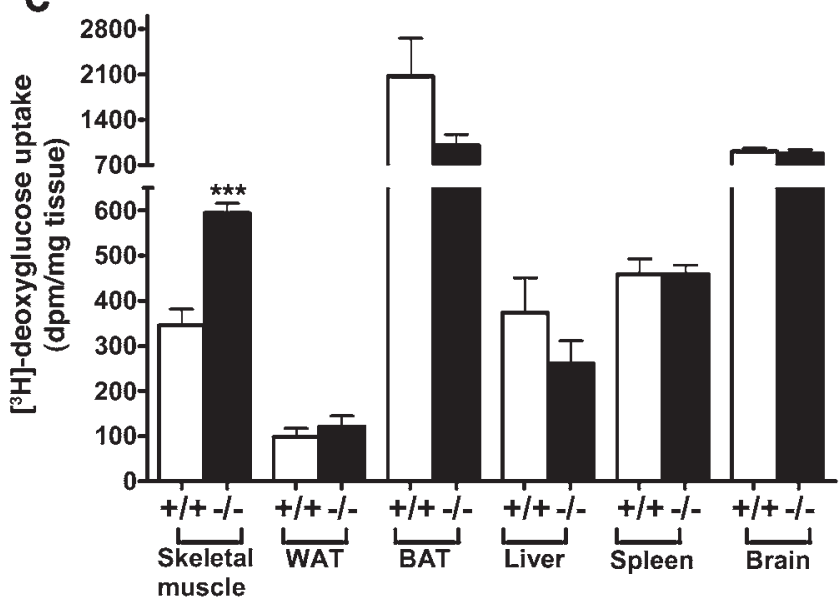

Figure 10

Improved glucose metabolism in inbred ad-Lrp1 $1^{-/-}$mice. (A) Fasting plasma glucose, insulin, and NEFA levels in chow-fed ad-Lrp1 $1^{+/+}$(white bars) and $a d-L r p 1^{-/-}$(black bars) mice. (B) Plasma glucose levels in inbred ad-Lrp $1^{+/+}$(open squares) and ad-Lrp $1^{-/-}$(filled circles) mice after 6 hours fasting followed by intraperitoneal injection of glucose $(2 \mathrm{~g} / \mathrm{kg} \mathrm{BW})$. Data represent mean \pm SEM from 8 mice per group. Integration of the data $(A \cup C)$ is shown in the inset. (C) Tissue distribution of 2-deoxy-[ $\left.{ }^{3} \mathrm{H}\right]$ glucose in inbred ad-Lrp1 ${ }^{1^{+/}}$and $a d-L r p 1^{-/}$mice after intravenous injection of 2-deoxy-[ $\left.{ }^{3} \mathrm{H}\right]$ glucose. Data represent mean \pm SEM from 5 mice in each group. ${ }^{*} P<0.05,{ }^{* *} P<0.005,{ }^{* \star *} P<0.0005$.

cient and requires increased caloric combustion. This interpretation of the data is also in line with the increased food intake of these mice. The increased oxygen consumption and energy expenditure displayed by ad-Lrp1 $1^{-/-}$mice, with their reduced BAT mass and impaired heat dissipation mechanism, is consistent with this hypothesis. These results are also consistent with the concept of muscle shivering as the first-order compensatory mechanism for impaired BAT functions in body temperature maintenance (45). These metabolic phenotypes observed in the ad-Lrp1 $1^{-/-}$mice, with alterations in the use of fat versus muscle tissues for body core temperature maintenance, closely parallel the metabolic phenotypes of $\mathrm{Plin}^{-1-}$ mice with targeted inactivation of the perilipin gene (48). The Plin ${ }^{-1}$ mice displayed constitutively active hormone-sensitive lipase activity in adipocytes, resulting in less fat storage and smaller adipocytes. Interestingly, the Plin $^{-1}$ mice also consumed more food, displayed increased sensitivity to the cold environment, and were more resistant to diet-induced obesity (48). Importantly, indirect calorimetry measurements revealed increased oxygen consumption by Plin $^{-1-}$ compared with wild-type mice. Moreover, muscle mass was also increased in the $\mathrm{Plin}^{-/-}$mice (48). Thus, the $\mathrm{Plin}^{-/-}$mice with defective lipid storage potential in both WAT and BAT also required increased energy utilization by the muscle to compensate for their ineffective BAT thermogenic mechanism in maintenance of body core temperature. Taken together, the metabolic pheno- types of ad-Lrp $1^{-/-}$and $\mathrm{Plin}^{-/-}$mice indicate that poor lipid storage in brown and white adipocytes, due to defective lipid uptake and storage mechanisms, respectively, results in shifting from BAT thermogenesis to the use of a less energy-efficient muscular activity mechanism to maintain body core temperature. This shift of tissue energy metabolism is protective against diet-induced obesity.

In addition to the reduction in BAT and WAT, the ad-Lrp1-/mice also displayed a marked improvement in whole-body glucose disposal compared with the ad-Lrp $1^{+/+}$mice. The determination of glucose uptake by individual tissues revealed that the skeletal muscle is the major tissue responsible for the elevated plasma glucose clearance. This difference in glucose uptake between skeletal muscle of $a d-L r p 1^{+/+}$and $a d-L r p 1^{-/-}$mice may reflect the increase in energy demand for muscle thermogenesis and the overall decrease in adipose fat storage in ad-Lrp $1^{-/-}$mice. Thus, this study showed that inactivation of adipose LRP1 activity not only reduces fat storage in both WAT and BAT and protects against diet-induced obesity, but that it also protects against glucose intolerance and diabetes due to increased energy expenditure shifting from brown adipose thermogenesis to muscular activity for maintenance of body temperature.

In summary, this study documents the prominent role of adipocyte LRP1 in modulating energy metabolism and sensitivity to diet-induced obesity and diabetes. These results add an impor- 
A
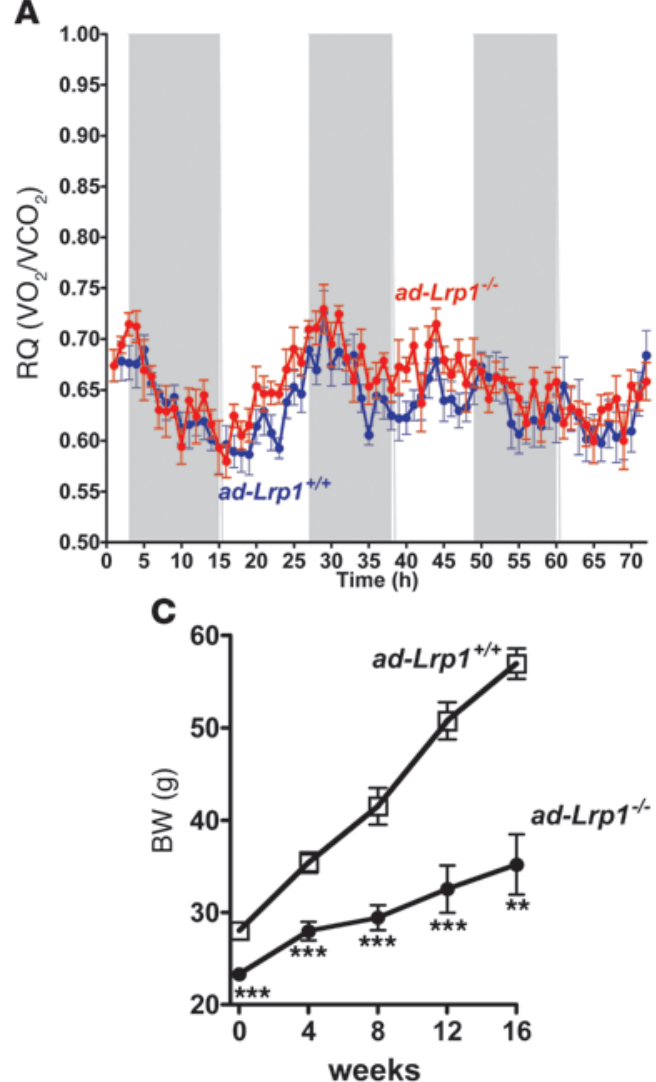

B

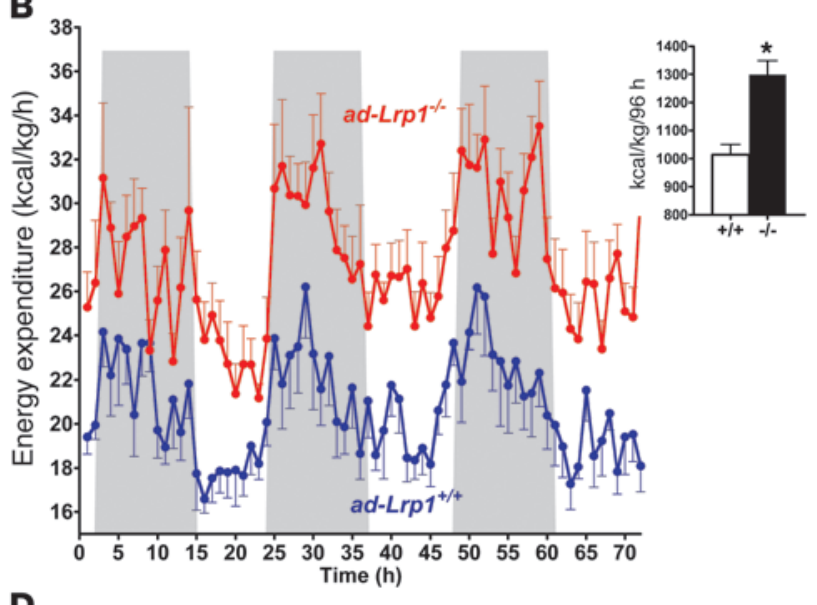

D

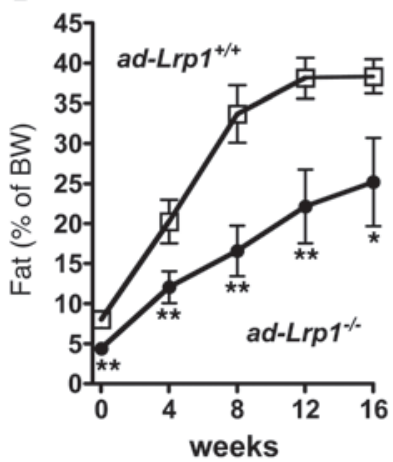

Figure 11

Resistance to diet-induced obesity of inbred ad-Lrp1-/- mice. Measurements of respiratory quotient $(\mathbf{A})$ and energy expenditure (B) in inbred ad-Lrp $1^{+/+}$(blue tracings) and ad-Lrp1-/- (red tracings) mice after high-fat feeding. The inset in B shows cumulative energy expenditure measurements from the respective tracings. (C and $\mathbf{D})$ Effects of high-fat diet on body fat gain and fat pad mass in inbred ad-Lrp1 ${ }^{+/+}$(open squares) and ad-Lrp1 ${ }^{-/-}$(filled circles) mice. ${ }^{*} P<0.05,{ }^{\star \star} P<0.005,{ }^{\star \star *} P<0.0005$.

tant perspective to the currently established models in showing the unique importance of various LDL receptor family proteins in these metabolic processes. Previously, VLDL receptor expression in adipocytes was shown to play a role in adiposity by modulating LpL-mediated lipid uptake (14). Additionally, LRP5 was shown to participate in glucose-induced insulin secretion by islets, with its defective expression causing impaired glucose tolerance (49). More recently, LRP6 mutations in humans have been implicated in the metabolic syndrome consisting of hyperlipidemia, hypertension, and diabetes via impairment of the Wnt signaling cascade (50). These continued investigations aimed at identifying the functional roles of each of the LDL receptor family proteins expressed in various tissues will add further insights into lipid transportdependent and -independent functions of these receptor proteins in physiology and pathophysiology of metabolic diseases.

\section{Methods}

Generation of ad-Lrp1 $1^{-/-}$mice. Two lines of adipose tissue-specific LRP1-knockout mice were generated independently at the University of Cincinnati College of Medicine and the University of Texas Southwestern Medical Center. For the generation of the ad-Lrp1 $1^{-/-}$mouse strain in Cincinnati, adiposespecific Cre recombinase transgenic mice in a C57BL/6 background were obtained from Ronald M. Evans (Salk Institute, La Jolla, California, USA) (23) and crossbred with C57BL/6 Lrp $1^{\text {flox flox }}$ mice (28). Mouse genotype was confirmed by PCR using previously described primers and thermocycler conditions $(23,28)$. The ad-Lrp1 $1^{-/-}$mouse colony in the Dallas laboratory was obtained by crossing aP2-Cre transgenic mice with $\operatorname{Lrp} 1^{\text {flox } / \text { flox }}$ mice. These ad-Lrp1 $1^{-1-}$ mice were maintained on a mixed B6/129 background. Genotyping was performed by PCR using 3 primers with the following sequences: sense: 5'-GGAAATGATCTGGCCCCCATTGGTCACTCC-3'; 2 antisense: $5^{\prime}$-CACTGGAGACAAGCTTCCAGGTTCCCACAA- $3^{\prime}$ in aP2 gene and $5^{\prime}$ CGCGAACATCTTCAGGTTCTGCGGGAAACC- $3^{\prime}$ in Cre transgene. PCR amplification began with a 1-minute denaturation step at $94^{\circ} \mathrm{C}$ and then 30 cycles of denaturation at $94^{\circ} \mathrm{C}$ for 30 seconds, annealing at $60^{\circ} \mathrm{C}$ for 1 minute, and extension at $72^{\circ} \mathrm{C}$ for 1 minute. The products were further analyzed by agarose gel electrophoresis to confirm the size.

Animals were housed in specific pathogen-free facilities with a 12-hour light/12-hour dark cycle. Animals were fed basal rodent chow 5058 PicoLab Mouse Diet 20 (9\% fat by weight; LabDiet) or a high-fat diet containing $35.8 \%$ fat by weight (D12330; Research Diets), and agematched male littermates were used for all experiments. All experimental protocols were approved by the Institutional Animal Care and Use Committees of the University of Cincinnati and University of Texas Southwestern Medical Center.

Preparation of mature adipocytes and stromal-vascular cell fractions from fat tissue. Epididymal and subcutaneous fat pads were isolated from mice by dissection $(n=3)$ and washed in Krebs-Ringer-bicarbonate buffer ( $\mathrm{pH} 7.4)$. The tissues were incubated with $0.1 \%$ collagenase (Worthington) on a shak- 
A

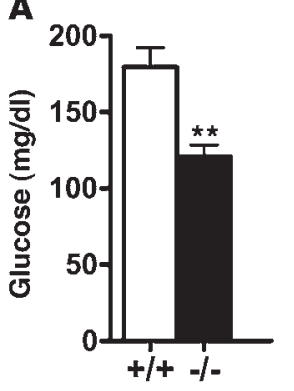

B

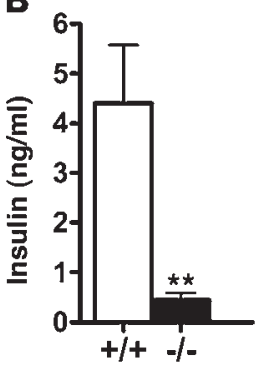

C

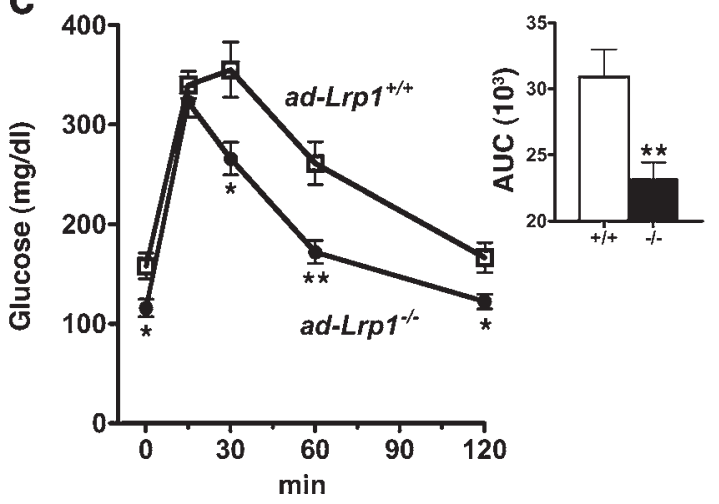

ing platform at $37^{\circ} \mathrm{C}$ for 20 minutes and then filtered through $100-\mu \mathrm{m}$ mesh to remove undigested tissue. After centrifugal separation at $200 \mathrm{~g}$ in a microfuge, mature adipocytes were collected from the top of the tubes, and stromal-vascular fractions were isolated from the bottom of the tubes. Both cell fractions were incubated overnight in DMEM containing 10\% FBS prior to homogenization in protease inhibitor-containing buffer for immunoblot analysis.

Plasma lipid clearance. Postprandial lipid clearance was determined by gavage feeding the animals $(n=10)$ with a bolus dose of olive oil $(15 \mu \mathrm{l} / \mathrm{g} \mathrm{BW})$ after a 6-hour fasting period. Blood samples were collected from the tail vein immediately before and at 1,2, and 3 hours after the oral bolus load. For experiments to determine lipolysis versus whole-particle uptake by various tissues, an Intralipid emulsion containing $1 \times 10^{6} \mathrm{dpm}$ of $\left[{ }^{3} \mathrm{H}\right]$ cholesteryl oleate (Amersham) and 500,000 dpm of $\left[{ }^{14} \mathrm{C}\right]$ trioleate (Amersham) was injected intravenously into each mouse as described previously (51). Blood was collected from the retroorbital plexus at 30 seconds and 1, 2, and 4 minutes after injection. At the 5 -minute time point, mice were perfused with cold saline, tissues removed, and plasma decay and tissue counts measured.

LPL activity determinations. Total cell extracts were prepared by homogenizing $90 \mathrm{mg}$ of tissues in buffer containing $20 \mathrm{mM}$ Tris- $\mathrm{HCl}$, pH 8.0, $1 \mathrm{mM}$ EDTA, and $300 \mu \mathrm{M}$ sodium taurocholate. Cell-surface LpL was isolated from the minced tissues by collecting supernatants from centrifugation of samples for 10 minutes at 20,000 $\mathrm{g}$ in a benchtop microfuge after incubation for 1 hour at $37^{\circ} \mathrm{C}$ in buffer containing $10 \mathrm{mM}$ Tris- $\mathrm{HCl}, \mathrm{pH} 7.4,10 \mathrm{U} / \mathrm{ml}$ heparin, and $0.5 \%$ heat-inactivated mouse serum. Total lipolytic activities in tissue homogenates and in the heparin-released supernatants were determined based on the hydrolysis of the fluorogenic substrate 4-methylumbelliferyl heptanoate (Sigma-Aldrich) in the presence or absence of $1 \mathrm{M} \mathrm{NaCl}$, as described previously (52). LpL-specific lipolytic activity was calculated from the differences observed in the presence or absence of $1 \mathrm{M} \mathrm{NaCl}$.

Body fat measurements. Body fat mass was measured in conscious mice $(n=16)$ using ${ }^{1} \mathrm{H}$ magnetic resonance spectroscopy (EchoMRI-100; Echomedical Systems). BW measurements were performed monthly for animals maintained on chow diet and weekly for animals maintained on the high-

\section{Figure 12}

Improved glucose metabolism in inbred ad-Lrp1 $1^{-/-}$mice fed a high-fat diet for 16 weeks. Fasting plasma glucose $(\mathbf{A})$ and insulin levels (B) in high fat diet-fed ad-Lrp1+/+ (white bars) and ad-Lrp1-/- (black bars) mice. (C) Plasma glucose levels in inbred ad-Lrp1+/+ (open squares) and ad-Lrp1-/- (filled circles) mice after 6 hours fasting followed by intraperitoneal injection of glucose (2 g/kg BW). Integration of the data (AUC) is shown in the inset in C. Data represent mean \pm SEM from 12 mice in each group. ${ }^{\star} P<0.05,{ }^{* \star} P<0.005$.

fat diet. After 1 week of adaptation, food intake (in grams of food) of individual mice was recorded daily for a week. For the food deprivation experiment, mice were fasted for 24 hours with water ad libitum. The ad-Lrp1 $1^{+/+}$ control group consisted of 16 mice, and the ad-Lrp1-/- experimental group had 18 mice. A BRUKER Minispec Time Domain Nuclear Magnetic Resonance (TD-NMR) analyzer was adopted to analyze fat/lean components of these live mice. The percentage of body fat was measured automatically by the TD-NMR analyzer. The reduction in the percentage of body fat after 24 hours of fasting was used as an indicator of fat loss.

Energy balance measurements. Animals $(n=16)$ were allowed to acclimate to respiratory chambers for a period of 2 days. Subsequently, energy expenditure (EE), respiratory quotient (RQ), food intake, and locomotive activity were measured simultaneously over 24-hour periods for 4 consecutive days using a customized 32-cage Indirect Calorimetry System combined with a Drinking and Feeding Monitor and TSE ActiMot system (TSE-Systems). The volume of oxygen consumed $\left(\mathrm{VO}_{2} ;[\mathrm{ml} /(\mathrm{kg} \times \mathrm{h})]\right)$ and carbon dioxide produced $\left(\mathrm{VCO}_{2}\right.$; $[\mathrm{ml} /(\mathrm{kg} \times \mathrm{h})]$ ) were measured to calculate $\mathrm{RQ}$ (ratio of $\mathrm{CO}_{2}$ produced to $\mathrm{VO}_{2}$ consumed $)$ and $\mathrm{EE}\left(\mathrm{EE}=[3.815+1.232 \times \mathrm{RQ}] \times \mathrm{VO}_{2} \times 0.001[\mathrm{kcal} /(\mathrm{kg} \times \mathrm{h})]\right)$.

Fine and locomotive activities. The TSE Indirect Calorimetry System was customized to include a multidimensional light beam and light sensor system, which records and localizes light beam breaks within the entire home cage environment of each mouse. The parallel beams were set up close enough so that no mouse could be in the calorimeter cage without at least 1 light beam being broken by its body. The motor activity-monitoring software of this system was programmed to interpret breaking of consecutive beams of the lower level of the beam grid to indicate ambulatory movement. Recorded breaks of light beams within a second beam grid located in the upper half of the home cage would be interpreted as rearing behavior, which often correlates with feeding from hanging feeders or climbing activities. Finally, rapid consecutive breaks and reconnection of the same light beams were interpreted as stationary motor activity or fidgeting. The recordings of such events were used to indicate shivering or fidgeting behavior.

Cold adaptation studies. Ten $\operatorname{ad}-\operatorname{Lrp} 1^{+/+}$and $10 \mathrm{ad}-\operatorname{Lrp} 1^{-/-}$mice were placed in a cold-temperature environment of $4^{\circ} \mathrm{C}$ and body core temperature measured using a rectal thermometer (Physitemp). Heat production in a subset of 6 mice from each group was visualized using a high-resolution infrared camera (FLIR PM280; FLIR Systems) that applies radiometry technology to convert infrared energy (radiant heat) into 12-bit dynamic range color images. The images were captured from the backs of the mice before and 1 hour after exposure to the $4^{\circ} \mathrm{C}$ environment. Infrared thermography images were taken from the upper half of the body to specifically visualize heat production from the BAT.

Glucose tolerance and tissue uptake. Blood samples were collected immediately before and at $15,30,60$, and 120 minutes after intraperitoneal injection of glucose $(2 \mathrm{~g} / \mathrm{kg} \mathrm{BW})$ into a 6 -hour-fasted animal. Tissue uptake of glucose was monitored by intravenous injection of glucose $(1 \mathrm{~g} / \mathrm{kg}$ BW) along with 2-deoxy $\left[{ }^{3} \mathrm{H}\right]$ glucose $(0.5 \mu \mathrm{Ci} / \mathrm{g}$ BW; NEN Life Science Products) after a 6-hour fast. Animals were euthanized after 15 minutes, extensively perfused with buffered saline, and tissues were excised, weighed, and solubilized with NCSR-II Tissue Solubilizer (Amersham). 
Radioactivity in each sample was quantified by liquid scintillation counting. Data are reported as total 2 -deoxy $\left[{ }^{3} \mathrm{H}\right]$ glucose radiolabel incorporated per milligram of tissue weight.

Blood chemistry. Animals were fasted for 6 hours prior to blood collection from the tail vein. Plasma triglyceride and cholesterol levels were determined using the Infinity Triglyceride and Cholesterol Kits (Thermo Electron Corp.). Blood glucose was determined with an Accu Check Active Glucometer (Roche Applied Science). Plasma insulin and leptin levels were measured in individual samples with multiplexed biomarker immunoassays for Luminex xMAP (Linco).

Histology. Tissues were fixed by immersion in neutral buffered formalin $(10 \%)$, dehydrated in ethanol and then transferred to xylene solution for embedding in paraffin. Five- and $8-\mu \mathrm{m}$ sections were stained with H\&E.

Quantitative real-time PCR. Total RNA from WAT (epididymal) and BAT was obtained using RNA STAT-60 (TEL-TEST Inc.). The extracted RNA was treated with DNAase (Ambion) before reverse transcription (Applied Biosystems). SYBR Green PCR Master Mix (Applied Biosystems) was used for quantitative real-time PCR. The sequences of 2 primers for LRP1 were: sense, 5'-GACCAGGTGTTGGACACAGATG-3'; antisense, 5'-AGTCGTTGTCTCCGTCACACTTC-3'. GAPDH was used as a control to normalize the amount of RNA sample. The sequence of its sense primer was 5'-TGTGTCCGTCGTGGATCTGA-3', and the antisense one was 5'-CCTGCTTCACCACCTTCTTGAT-3'.

Immunoblot analysis. The expression of LRP1 in mice from Dallas was analyzed by preparing membrane fractions from isolated white (epididymal) and brown fat pads. Briefly, the adipose pads were homogenized in buffer $\mathrm{A}$ (20 mM Tris-HCl, pH 8.0, $1 \mathrm{mM} \mathrm{CaCl}_{2}, 150 \mathrm{mM} \mathrm{NaCl}$ ) plus fresh protease inhibitors (P8430; Sigma-Aldrich) followed by centrifugation at $800 \mathrm{~g}$ for 5 minutes at $4^{\circ} \mathrm{C}$. The supernatant was then centrifuged at $100,000 \mathrm{~g}$ for 30 minutes at $4^{\circ} \mathrm{C}$. The membrane pellet was resuspended in buffer $\mathrm{B}$ (50 mM Tris-HCl, pH 8.0, $2 \mathrm{mM} \mathrm{CaCl}_{2}, 80 \mathrm{mM} \mathrm{NaCl}, 1 \%$ Triton X-100). After another centrifugation at $100,000 \mathrm{~g}$ for 30 minutes at $4{ }^{\circ} \mathrm{C}$, the supernatant was collected for immunoblotting. The membrane extract was resolved on SDS-polyacrylamide gel and transferred to nitrocellulose membranes (Hybond-C Extra; Amersham Biosciences). The blot was blocked with $5 \%$ skim milk, probed with rabbit anti-LRP1 antibody, and incubated with HRP-conjugated goat anti-rabbit secondary antibody (Amersham Biosciences). Immunoreactive bands were visualized using a chemiluminescence detection kit (Amersham Biosciences).

Immunoblot analysis conducted in Cincinnati was performed by enriching LRP1 in tissue extracts by precipitation with recombinant receptor-associated protein (RAP) conjugated to glutathione-S-transferase in the presence of glutathione beads. The precipitated proteins were separated by SDS-PAGE, transferred to polyvinylidene fluoride paper, and blotted with antibodies against the $\beta$ subunit of LRP1 (11H4; ATCC). Expression of UCP-1, -2, and -3 in tissues was analyzed by immunoblotting tissue homogenates with UCP-1 and UCP- 2 antibodies obtained from Calbiochem and UCP-3 antibodies from Alpha Diagnostics. Immunoreactive proteins were detected by incubating the blots with fluorescently labeled species-specific secondary antibodies (Molecular Probes; Invitrogen) and visualized by the Odyssey Infrared Imaging System (Li-Cor Biosciences).

Statistics. Numerical data are expressed as mean \pm SEM. An unpaired 2tailed Student's $t$ test was used for analysis of 1 -group variables. A $P$ value of less than 0.05 between the groups was considered significant.

\section{Acknowledgments}

We are grateful to Ronald Evans for providing the aP2-Cre recombinase transgenic mice for this study. This research was supported by grants DK74932 (to D.Y. Hui), DK069987 and DK056863 (to M.H. Tschöp), and HL20948 and HL63762 (to J. Herz) from the NIH. S.M. Hofmann is the recipient of a Scientist Development Award $(0635079 \mathrm{~N})$ from the National Center of the American Heart Association. D. Gilham is the recipient of a Post-Doctoral Fellowship (0625329B) from the Ohio Valley Affiliate of the American Heart Association. J. Herz is an Established Investigator of the American Heart Association.

Received for publication February 22, 2007, and accepted in revised form August 1, 2007.

Address correspondence to: David Y. Hui, Department of Pathology, University of Cincinnati Genome Research Institute, 2120 E. Galbraith Road, Cincinnati, Ohio 45237, USA. Phone: (513) 558-9152; Fax: (513) 558-1312; E-mail: huidy@email.uc.edu. Or to: Joachim Herz, Department of Molecular Genetics, University of Texas Southwestern Medical Center, 5323 Harry Hines Blvd., Dallas, Texas 75390, USA. Phone: (214) 648-5633; Fax: (214) 648-8804; E-mail: joachim.herz@utsouthwestern.edu. Or to: Matthias Tschöp, Department of Psychiatry, University of Cincinnati Genome Research Institute, 2120 E. Galbraith Road, Cincinnati, Ohio 45237, USA. Phone: (513) 558-8648; Fax: (513) 558-8656; E-mail: tschoemh@ucmail.uc.edu.

Susanna M. Hofmann, Li Zhou, and Diego Perez-Tilve contributed equally to this work and are co-first authors.
1. Boden, G. 1997. Role of fatty acids in the pathogenesis of insulin resistance and NIDDM. Diabetes. 46:3-10.

2. Randle, P.J. 1998. Regulatory interactions between lipids and carbohydrates: The glucose fatty acid cycle after 35 years. Diabetes Metab. Rev. 14:263-283.

3. Havel, P.J. 2004. Regulation of energy balance and carbohydrate/lipid metabolism. Diabetes. 53(Suppl. 1):S143-S151.

4. Boden, G., Lebed, B., Schatz, M., Homko, C., and Lemieux, S. 2001. Effects of acute changes of plasma free fatty acids on intramyocellular fat content and insulin resistance in healthy subjects. Diabetes. 50:1612-1617.

5. Hajri, T., Han, X.X., Bonen, A., and Abumrad, N.A. 2002. Defective fatty acid uptake modulates insulin responsiveness and metabolic responses to diet in CD36-null mice. J. Clin. Invest. 109:1381-1389. doi:10.1172/JCI200214596.

6. Febbraio, M., et al. 1999. A null mutation in murine CD36 reveals an important role in fatty acid and lipoprotein metabolism. J. Biol. Chem. 274:19055-19062.

7. Mahley, R.W., and Ji, Z.S. 1999. Remnant lipoprotein metabolism: Key pathways involving cell-surface heparan sulfate proteoglycans and apolipoprotein E. J. Lipid Res. 40:1-16.

8. Schreyer, S.A., Vick, C., Lystig, T.C., Mystkowski, P., and LeBoeuf, R.C. 2002. LDL receptor but not apolipoprotein E deficiency increases diet-induced obesity and diabetes in mice. Am. J. Physiol. Endocrinol. Metab. 282:E207-E214.

9. Pedrini, M.T., et al. 2005. Human triglyceride-rich lipoproteins impair glucose metabolism and insulin signalling in L6 skeletal muscle cells independently of non-esterified fatty acid levels. Diabetologia. 48:756-766.

10. Dergunov, A.D., et al. 2000. Conformation of apolipoprotein $\mathrm{E}$ both in free and in lipid-bound form may determine the avidity of triglyceride-rich lipoproteins to the LDL receptor: structural and kinetic study. Biochim. Biophys. Acta. 1484:14-28.

11. Dergunov, A.D., et al. 2000. Structural peculiarities of the binding of very low density lipoproteins and low density lipoproteins to the LDL receptor in hypertriglyceridemia: role of apolipoprotein E. Biochim. Biophys. Acta. 1484:29-40.

12. Rensen, P.C.N., et al. 1997. Particle size determines the specificity of apolipoprotein E-containing triglyceride-rich emulsions for the LDL receptor versus hepatic remnant receptor in vivo. J. Lipid Res. 38:1070-1084.

13. Kobayashi, K., et al. 1996. Reversal of hypercholesterolemia in low density lipoprotein receptor knockout mice by adenovirus-mediated gene transfer of the very low density lipoprotein receptor. J. Biol. Chem. 271:6852-6860.

14. Yagyu, H., et al. 2002. Very low density lipoprotein (VLDL) receptor-deficient mice have reduced lipoprotein lipase activity. Possible causes of hypertriglyceridemia and reduced body mass with VLDL receptor deficiency. J. Biol. Chem. 
277:10037-10043.

15. Kowal, R.C., Herz, J., Goldstein, J.L., Esser, V., and Brown, M.S. 1989. Low density lipoprotein receptorrelated protein mediates uptake of cholesteryl esters derived from apoprotein E-enriched lipoproteins. Proc. Natl. Acad. Sci. U. S. A. 86:5810-5814.

16. Kowal, R.C., et al. 1990. Opposing effects of apolipoproteins $\mathrm{E}$ and $\mathrm{C}$ on lipoprotein binding to low density lipoprotein receptor-related protein. J. Biol. Chem. 265:10771-10779.

17. Chappell, D.A., et al. 1993. Lipoprotein lipase induces catabolism of normal triglyceride-rich lipoproteins via the low density lipoprotein receptor-related protein/alpha2-macroglobulin receptor in vitro. J. Biol. Chem. 268:14168-14175.

18. Nykjaer, A., et al. 1993. The alpha2-macroglobulin receptor/low density lipoprotein receptor related protein binds lipoprotein lipase and $\beta$-migrating very low density lipoprotein associated with the lipase. J. Biol. Chem. 268:15048-15055.

19. Merkel, M., et al. 1998. Catalytic inactive lipoprotein lipase expression in muscle of transgenic mice increases very low density lipoprotein uptake: Direct evidence that lipoprotein lipase bridging occurs in vivo. Proc. Natl. Acad. Sci. U. S. A. 95:13841-13846

20. Goudriaan, J.R., et al. 2001. Protection from obesity in mice lacking the VLDL receptor. Arterioscler. Thromb. Vasc. Biol. 21:1488-1493.

21. Descamps, O., Bilheimer, D., and Herz, J. 1993. Insulin stimulates receptor-mediated uptake of apoE-enriched lipoproteins and activated alpha 2 -macroglobulin in adipocytes. J. Biol. Chem. 268:974-981.

22. Bluher, M., et al. 2002. Adipose tissue selective insulin receptor knockout protects against obesity and obesity-related glucose intolerance. Dev. Cell. 3:25-38.

23. He, W., et al. 2003. Adipose-specific peroxisome proliferator-activated receptor \{gamma\} knockout causes insulin resistance in fat and liver but not in muscle. Proc. Natl. Acad. Sci. U. S. A. 100:15712-15717.

24. Hotamisligil, G.S., et al. 1996. Uncoupling of obesity from insulin resistance through a targeted mutation in aP2, the adipocyte fatty acid binding protein. Science. 274:1377-1379.

25. Shimomura, I., et al. 1998. Insulin resistance and diabetes mellitus in transgenic mice expressing nuclear SREBP-1c in adipose tissue: Model for congenital generalized lipodystrophy. Genes Dev. 12:3182-3194.

26. Tozzo, E., Shepherd, P.R., Gnudi, L., and Kahn,
B.B. 1995. Transgenic GLUT-4 overexpression in fat enhances glucose metabolism: Preferential effect on fatty acid synthesis. Am. J. Physiol. 268:E956-E964.

27. Zhang, Y., et al. 1994. Positional cloning of the mouse obese gene and its human homologue. Nature. 372:425-432.

28. Rohlmann, A., Gotthardt, M., Willnow, T.E., Hammer, R.E., and Herz, J. 1996. Sustained somatic gene inactivation by viral transfer of cre recombinase. Nat. Biotechnol. 14:1562-1565.

29. Yamauchi, T., et al. 2001. The mechanisms by which both heterozygous peroxisome proliferatoractivated receptor $\gamma$ (PPAR $\gamma$ ) deficiency and PPAR $\gamma$ agonist improve insulin resistance. J. Biol. Chem. 276:41245-41254.

30. Chen, H.C., et al. 2002. Increased insulin and leptin sensitivity in mice lacking acyl CoA:diacylglycerol acyltransferase 1. J. Clin. Invest. 109:1049-1055. doi:10.1172/JCI200214672.

31. Voshol, P.J., et al. 2003. Increased hepatic insulin sensitivity together with decreased hepatic triglyceride stores in hormone-sensitive lipase-deficient mice. Endocrinology. 144:3456-3462.

32. Zhang, J., et al. 2004. Selective disruption of PPAR $\gamma 2$ impairs the development of adipose tissue and insulin sensitivity. Proc. Natl. Acad. Sci. U. S. A. 101:10703-10708.

33. Lee, K., et al. 2003. Inhibition of adipogenesis and development of glucose intolerance by soluble preadipocyte factor-1 (pref-1). J. Clin. Invest. 111:453-461. doi:10.1172/JCI200315924.

34. Willnow, T.E., and Herz, J. 1994. Genetic deficiency in low density lipoprotein receptor-related protein confers cellular resistance to pseudomonas exotoxin A. J. Cell Sci. 107:719-726.

35. Zhang, H., et al. 2004. Localization of low density lipoprotein receptor-related protein 1 to caveolae in 3T3-L1 adipocytes in response to insulin treatment. J. Biol. Chem. 279:2221-2230.

36. Aihaud, G., Grimaldi, P., and Negrel, R. 1992. Cellular and molecular aspects of adipose tissue development. Annu. Rev. Nutr. 12:207-233.

37. Goldberg, I. 1996. Lipoprotein lipase and lipolysis: Central roles in lipoprotein metabolism and atherogenesis. J. Lipid Res. 37:693-707.

38. Ishibashi, S., Herz, J., Maeda, N., Goldstein, J.L., and Brown, M.S. 1994. The two receptor model of lipoprotein clearance: Tests of the hypothesis in knockout mice lacking the low density lipoprotein receptor, apolipoprotein E, or both proteins. Proc. Natl. Acad. Sci. U. S. A. 91:4431-4435

39. Rohlmann, A., Gotthardt, M., Hammer, R.E., and
Herz, J. 1998. Inducible inactivation of hepatic LRP gene by cre-mediated recombination confirms role of LRP in clearance of chylomicron remnants. J. Clin. Invest. 101:689-695.

40. Hussain, M.M., et al. 1991. Clearance of chylomicron remnants by the low density lipoprotein receptor-related protein $/ \alpha 2$-macroglobulin receptor. J. Biol. Chem. 266:13936-13940.

41. Chappell, D.A., et al. 1992. The low density lipoprotein receptor-related protein/ $\alpha 2$-macroglobulin receptor binds and mediates catabolism of bovine milk lipoprotein lipase. J. Biol. Chem. 267:25764-25767.

42. Chappell, D.A., et al. 1994. Cellular catabolism of normal very low density lipoproteins via the low density lipoprotein receptor-related protein/ $\alpha 2$ macroglobulin receptor is induced by the C-terminal domain of lipoprotein lipase. J. Biol. Chem. 269:18001-18006.

43. Véniant, M.M., et al. 1998. Lipoprotein clearance mechanisms in LDL receptor-deficient apoB48only and apoB100-only mice. J. Clin. Invest. 102:1559-1568.

44. Lowell, B.B., and Spiegelman, B.M. 2000. Towards a molecular understanding of adaptive thermogenesis. Nature. 404:652-660.

45. Himms-Hagen, J. 1989. Brown adipose tissue thermogenesis and obesity. Prog. Lipid Res. 28:67-115.

46. Lowell, B.B., et al. 1993. Development of obesity in transgenic mice after genetic ablation of brown adipose tissue. Nature. 366:740-742.

47. Zhou, Z., et al. 2003. Cidea-deficient mice have lean phenotype and are resistant to obesity. Nat. Genet. 35:49-56.

48. Martinez-Botas, J., et al. 2000. Absence of perilipin results in leanness and reverses obesity in lepr $(\mathrm{db} /$ db) mice. Nat. Genet. 26:474-479.

49. Fujino, T., et al. 2003. Low-density lipoprotein receptor-related protein $5(\operatorname{lrp} 5)$ is essential for normal cholesterol metabolism and glucose-induced insulin secretion. Proc. Natl. Acad. Sci. U. S. A. 100:229-234.

50. Mani, A., et al. 2007. Lrp6 mutation in a family with early coronary disease and metabolic risk factors. Science. 315:1278-1282

51. Augustus, A.S., Kako, Y., Yagyu, H., and Goldberg, I.J. 2003. Routes of fat delivery to cardiac muscle: Modulation of lipoprotein lipolysis alters uptake of TG-derived FA. Am. J. Physiol. Endocrinol. Metab. 284:E331-E339.

52. Gilham, D., and Lehner, R. 2005. Techniques to measure lipase and esterase activity in vitro. Methods. 36:139-147. 